\title{
Power Flow Analysis of the Enhanced Proposed 330kV Transmission Network of the Nigeria Grid
}

\author{
FESTUS OSAZEE AGBONTAEN*, JOEL OSARUMWENSE EGWAILE*, AND IGNATIUS KEMA OKAKWU*
}

\section{RECEIVED N 25.03.2019 ACCEPTED ON 03.05.2019}

\begin{abstract}
The Nigeria's power sector transmission infrastructure continues to be challenged as it still remains the weak link in the electricity supply chain. The Nigerian Federal Government on its Roadmap for power sector reform highlighted that to accommodate the planned increase in generation capacity, there was need for a $30 \%$ increase in the "true deliverable" transmission capacity of the country's $330 \mathrm{kV}$ network. But the technical feasibility of this plan is an issue to be considered.

In this work, the existing $330 \mathrm{kV}$ Nigeria transmission network was expanded by the introduction of new lines and power stations, simulation was carried out and the effect was analyzed using Newton-Raphson algorithm in ETAP 12.6. The base case operating condition as obtained from the power flow on which the various transfer cases were implemented, gives a fair generation and loading pattern of the Nigerian grid. The total installed generating capacity of the base case considered was 11,948MW out of which 4,347.21 MW was available for load level of 3,633.6MW. Result shows that the maximum load ability of the enhanced network was increased to $238.4 \%$ compared with the existing network when the NewtonRaphson iteration method was applied.
\end{abstract}

Key Words: Transmission Line, Network, Loadability, Load Flow, Maximum Load, Power Station.

\section{INTRODUCTION}

$\int$ ectrical power systems are the backbone of any developing modern society, supporting several other critical infrastructures, such as transportation, communication, water, etc. [1]. However, increase in economic activities resulting from increase in population and social advancement has led to increase in electrical energy demand. This has increased the burden on the existing transmission assets and in some cases, has caused the loading of the transmission assets beyond their design limits with consequent reduction in power quality and power outages in extreme cases [2].

The current transmission system in Nigeria comprises $5523.8 \mathrm{~km}$ of $330 \mathrm{kV}, 6801.49 \mathrm{~km}$ of $132 \mathrm{kV}, 32 \mathrm{No} .330 / 132$ $\mathrm{kV}$ substations with total installed transformer capacity of 7688 MVA. 105 No. 132/33/11 kV substations with total installed transformer capacity of $9130 \mathrm{MVA}$. The average available capacity on $330 / 132 \mathrm{kV}$ is 7364 MVA and 8448

Authors E-Mal: (festus.felix2326@gmail.com, egwaile.joel@uniben.edu, igokakwu@yahoo.com)

* Department of Electrical/Electronic Engineering, University of Benin, Benin City, Nigeria.

This is an open access article published by Mehran University Research Journal of Engineering and Technology, Jamshoro under the CC by 4.0 International License. 
MVA on $132 / 33 \mathrm{kV}$ [3]. Some of the major challenges associated with the South-South, South East and South West regions (Edo, Delta, Bayelsa, Rivers, AkwaIbom, Cross River, Abia, Enugu, Anambra, Imo, Ebonyi, Oyo, Osun, Ekiti, Ondo and Ogun States) of the transmission network are over voltages due to reactive power generation from the various power plants within the vicinity. And power is stranded due to the proximity of most of the gas thermal power stations and inadequacy of the tie lines to evacuate load. Lagos (Lagos State) south west region is prone to transmission congestion [3-4]. The grid bus is characterized by poor voltage profile in most parts of the network, frequent system collapse and exceedingly high transmission losses [5-6]. Furthermore, many electric networks in developing countries are facing the problem of voltage drop and energy loss, deterioration and overloaded with unbalanced loads [7]. Thus, there is need to have a sustainable, reliable and stable supply to create an enabling balanced economy [8]. The unreliability of the System Network has impacted on the nation's socioeconomic development and industrialization hence, the recent launch of the roadmap of the power sector reform by the Federal Government [9].

In order to increase power, there is a need to use all our sustainable energy sources like hydro, gas, wind, solar, and coal [10]. The reform also sought the maximization of access to electricity services, by promoting and facilitating consumer connections to the network systems in both rural and urban areas [11]. Energy planning experts using modern energy modeling tools estimate that for the Nigerian economy to grow at a rate of $10 \%$ the country's electricity requirement by 2020 will be of the order of 30,000 MW, and by 2030 it will be 78,000 MW [12].

\section{METHODOLOGY}

Data was collected from TCN (Transmission Company of Nigeria) for load flow analysis. The analysis on the data was done using Newton - Raphson algorithm in ETAP 12.6 load flow analyzer which is a power system modeling, design, analysis, optimization, control, and automation software.

Newton - Raphson method for load flow studies was used to simulate and investigate the generated power of the network. The load flow program calculates the magnitude and phase angle of the voltage at each bus, real and reactive power flowing in each line respectively.

A number of methods for power flow calculation use equations in different forms considering sending end $\left(\mathrm{i}^{\text {th }}\right.$ end) or receiving end ( $j^{\text {th }}$ end). In this work, we used the same method in section 2.26 of [13] where a detailed explanation of Newton-Raphson method as applicable to power flow problem was given.

The linear equation in compact form used is given as:

$$
\left(\begin{array}{c}
\Delta \mathrm{P} \\
\Delta \mathrm{Q}
\end{array}\right)=\left(\begin{array}{ll}
\mathrm{J}_{1} & \mathrm{~J}_{2} \\
\mathrm{~J}_{3} & \mathrm{~J}_{4}
\end{array}\right)\left(\begin{array}{c}
\Delta \delta \\
\Delta \mathrm{V}
\end{array}\right)
$$

Enhancement of the network was done based on the transmission planning region: South - East region (Anambra, Imo, Rivers, Akwa Ibom, Abia, Cross River, Enugu and Ebonyi), South - South region (Edo, Delta and Bayelsa), South - West region (Kwara, Oyo, Osun, Ekiti, Ondo and Ogun), Lagos region, North - West Region (Sokoto, Kebbi and Zamfara), North Region (Kastina, Jigawa and Kano), North - East Region (Yoba, Bornu, Gombe, Bauchi, Plateau and Taraba) and Central Region (Abuja, Kaduna, Niger, Nasarawa and Kogi) [14]. 


\section{PRESENTATION OF SYSTEM DATA}

The data used in this work is as presented in Tables 12. Twenty (20) generating stations, Sixty-Seven (67) transmission lines and a total of Fifty-Nine (59) buses stations with Thirty (30) load buses were considered for the existing network. The network was further expanded by adding the transmission lines presented in Table 3.

TABLE 1. EXISTING 330KV TRANSMISSION LINE NETWORK CIRCUIT

\begin{tabular}{|c|c|c|c|c|c|c|c|c|c|}
\hline No. & $\begin{array}{l}\text { From Station } \\
\text { Location }\end{array}$ & To Station Location & $\begin{array}{c}\text { Number of } \\
\text { Circuits }\end{array}$ & $\begin{array}{l}\text { Length } \\
(\mathrm{Km})\end{array}$ & No. & $\begin{array}{l}\text { From Station } \\
\text { Location }\end{array}$ & To Station Location & $\begin{array}{l}\text { Number of } \\
\text { Circuits }\end{array}$ & $\begin{array}{c}\text { Length } \\
(\mathrm{Km})\end{array}$ \\
\hline 1. & Afam GS & Alaoji T.S & 2 & 25 & 35. & Ikot - Ekpene T.S & Alaoji T.S & 2 & 57.9 \\
\hline 2. & Afam GS & PH main T.S & 2 & 8 & 36. & Ikot - Ekpene T.S & Odukpani T.S & 2 & 88.5 \\
\hline 3. & Ahoda T.S & Gbarian GS & 2 & 87.4 & 37. & Jebba G.S & Jebba T.S & 2 & 8 \\
\hline 4. & Aiyede T.S & Olorunsogo G.S & 1 & 125 & 38. & Jebba T.S & Ganmo T.S & 1 & 70 \\
\hline 5. & Ajah T.S & Lekki T.S & 2 & 5.5 & 39. & Jebba T.S & Osogbo T.S & 2 & 157 \\
\hline 6. & $\overline{\text { Ajah T.S }}$ & Alagbon T.S & 2 & 21 & 40. & Jebba T.S & Shiroro GS & 2 & 244 \\
\hline 7. & Ajaokuta T.S & Geregu GS & 1 & 75 & 41. & Jos T.S & Makurdi T.S & 1 & 285 \\
\hline 8. & Aladja T.S & Sapele G.S & 1 & 93 & 42. & Kaduna T.S & Shiroro GS & 2 & 95 \\
\hline 9. & Alagbon T.S & Lekki T.S & 2 & 24.5 & 43. & Kaduna T.S & Kano T.S & 1 & 230 \\
\hline 10. & Alaoji G.S & Alaoji T.S & 2 & 50 & 44. & Kaduna T.S & Jos T.S & 1 & 197 \\
\hline 11. & Alaoji T.S & Owerri T.S & 2 & 77.1 & 45. & Kainji T.S & Kainji G.S & 2 & 0.47 \\
\hline 12. & Asaba T.S & Onitsha T.S & 2 & 65.8 & 46. & Kainji T.S & Jebba T.S & 2 & 81 \\
\hline 13. & Benin T.S & Ajaokuta T.S & 2 & 195 & 47. & Katampe T.S & Gwagwalada T.S & 1 & 60 \\
\hline 14. & Benin T.S & Delta GS & 1 & 107 & 48. & lokoja T.S & Ajaokuta T.S & 1 & 215 \\
\hline 15. & Benin T.S & Sapela G.S & 2 & 50 & 49. & New Haven T.S & Ugwaji T.S & 2 & 65 \\
\hline 16. & Benin T.S & Asaba T.S & 2 & 153.9 & 50. & New Haven T.S & Onitsha T.S & 1 & 96 \\
\hline 17. & Birnin - Kebbi T.S & Kainji T.S & 1 & 310 & 51. & Odukpani T.S & Adiabor T.S & 2 & 10 \\
\hline 18. & Damaturu T.S & Maiduguri T.S & 1 & 260 & 52. & Okearo T.S & Egbin T.S & 2 & 30 \\
\hline 19. & Delta GS & Aladja T.S & 1 & 30 & 53. & Omotosho GS & Benin T.S & 1 & 120 \\
\hline 20. & Egbin GS & Benin T.S & 1 & 218 & 54. & Onitsha T.S & Okpai GS & 2 & 56 \\
\hline 21. & Egbin GS & Aja T.S & 2 & 14 & 55. & Onitsha T.S & Alaoji T.S & 1 & 138 \\
\hline 22. & Ganmo T.S & Osogbo T.S & 1 & 87 & 56. & Osogbo T.S & Aiyede T.S & 1 & 119 \\
\hline 23. & Gbarian G.S & Yenegoa T.S & 2 & 25 & 57. & Osogbo T.S & Ikeja west T.S & 1 & 235 \\
\hline 24. & Gombe T.S & Damaturu T.S & 1 & 160 & 58. & Osogbo T.S & Ihovbor G.S & 1 & 251 \\
\hline 25. & Gombe T.S & Yola T.S & 1 & 240 & 59. & Owerri T.S & Ahoda T.S & 2 & 73.6 \\
\hline 26. & Gwagwalada & Shiroro G.S & 1 & 144 & 60. & PH main T.S & Omoko T.S & 2 & 83 \\
\hline 27. & Gwagwalada T.S & Lokoja T.S & 1 & 135 & 61. & Sapele G.S & Delta G.S & 1 & 93 \\
\hline 28. & Ihovbor G.S & Benin T.S & 1 & 20 & 62. & Shiroro G.S & Katampe T.S & 1 & 144 \\
\hline 29. & Ikeja west T.S & Akangba T.S & 2 & 18 & 63. & Paras energy T.S & Sagamu T.S & 2 & 4 \\
\hline 30. & Ikeja west T.S & Omotosho T.S & 1 & 160 & 64. & Trans amadi T.S & PH main T.S & 2 & 8 \\
\hline 31. & Ikeja west T.S & Okearo T.S & 2 & 32 & 65. & Egbin GS & Ikorodu T.S & 2 & 17 \\
\hline 32. & Ikeja west T.S & Egbin T.S & 1 & 62 & 66. & Ikorodu T.S & Sagamu T.S & 2 & 34 \\
\hline 33. & Ikeja west T.S & Olorunsogo G.S & 1 & 50 & 67. & Asco GS & Ajaokuta T.S & & 50 \\
\hline 34. & Ikot - Ekpene T.S & Ugwaji T.S & 2 & 174 & \multicolumn{4}{|c|}{ Total } & 6717.67 \\
\hline
\end{tabular}

Mehran University Research Journal of Engineering \& Technology, Volume 38, No. 4, October, 2019 [p-ISSN: 0254-7821, e-ISSN: 2413-7219] 


\section{DISCUSSION OF RESULT FROM THE ANALYZED NETWORK.}

The maximum load the existing network when subjected to various loading conditions is $4,948.78 \mathrm{MW}$ and 1,448.72MVar for a base load of 4,347.21MW and 738.978MVar with losses of 246.253MW. And Damaturu T.S (0.82p.u), Gombe T.S (0.82p.u), Jos T.S (0.84p.u), Kaduna T.S (0.81p.u), Kano T.S (0.81p.u), Katempa T.S (0.82p.u) and Yola T.S (0.81p.u) violated the statutory voltage limit of $0.85 \mathrm{p} . \mathrm{u}-1.05 \mathrm{p} . \mathrm{u}$ [15]. However, the expanded network according to the transmission planning region was simulated and analyzed to determine the performance effect when enhanced to allow more power to be evacuated within the network.

The load carrying capacity of the network was determined by using ETAP 12.6 Load flow Analyzer to simulate the $330 \mathrm{kV}$ expanded/proposed network consisting of seventy-six (76) buses, ninety-three (93) transmission lines and thirty (30) generating stations $(18,975 \mathrm{MW}$ installed generating capacity). The input data used in the simulation of the maximum load capacity include various line loading and generation as obtained from TCN which represents the base case for the model.

TABLE 2. BUS LOADING

\begin{tabular}{|c|c|c|}
\hline No. & Terminal Bus & Rating (MVA) \\
\hline 1. & Afam & 105.882 \\
\hline 2. & Aiyede T.S & 117.647 \\
\hline 3. & Aja T.S & 58.824 \\
\hline 4. & Ajaokuta T.S & 117.647 \\
\hline 5. & Akangba T.S & 131.765 \\
\hline 6. & Aladja T.S & 96.588 \\
\hline 7. & Alaoji T.S & 117.647 \\
\hline 8. & Alaoji G.S & 52.588 \\
\hline 9. & Benin T.S & 184.471 \\
\hline 10. & Birni-Kebbi T.S & 117.647 \\
\hline 11. & Delta & 75.647 \\
\hline 12. & Egbin G.S & 1176.471 \\
\hline 13. & Ganmo T.S & 75.882 \\
\hline 14. & Geregu NIPP \& GS & 164.706 \\
\hline 15. & Gombe T.S & 235.294 \\
\hline 16. & Ikeja West T.S & 352.941 \\
\hline 17. & Jebba T.S & 10.118 \\
\hline 18. & Jebba G.S & 117.647 \\
\hline 19. & Jos T.S & 352.941 \\
\hline 20. & Kaduna T.S & 195.529 \\
\hline 21. & Kainji G.S & 117.647 \\
\hline 22. & Kano T.S & 217.529 \\
\hline 23. & Katampe T.S & 235.294 \\
\hline 24. & Maiduguri T.S & 58.824 \\
\hline 25. & New Haven T.S & 294.118 \\
\hline 26. & Okaero T.S & 117.647 \\
\hline 27. & Okpia G.S & 11.765 \\
\hline 28. & Onitsha T.S & 135.529 \\
\hline 29. & Oshogbo T.S & 176.471 \\
\hline 30. & Shiroro G.S & 72.353 \\
\hline 31. & Yola T.S & 117.647 \\
\hline
\end{tabular}


Electric power transfer was investigated by the insertion of the following power station; Calabar (561MW), Ibom (191MW), Aba (140MW), Azura(450MW), Kano (500MW), Mambilla (3,050MW), Zungeru (700MW), Itobe (1,200MW), Kiri (35MW) and Zamfara (100MW) into the network which increased the total installed capacity from 11,948-18,975MW (i.e. 58.81\% increase). The network was further expanded by adding the transmission lines shown in Table 3 to ascertain the maximum load carrying capacity of the network.
Results show that the network was able to evacuate 16,746.859MW (238.4\% maximum loadability increase) and 8,025.291MVar with loading of $16,400.067 \mathrm{MW}$ and 10,163.847MVar and Dandikowa T.S (0.80p.u) was out of the statutory voltage limit of $0.85-1.05 \mathrm{p}$.u. as seen in the power flow result in Table 4 and voltage profile diagram presented in Fig. 1. However, it was observed that the total losses on the transmission network is $252.25 \mathrm{MW}$ as shown in the line flow result presented in Table 5. Fig. 2, shows section of the simulation diagram of the network modeled on ETAP 12.6.

TABLE 3. TRANSMISSION LINES ADDED TO THE EXISTING NETWORK

\begin{tabular}{|c|c|c|c|}
\hline No. & Transmission Line & $\begin{array}{c}\text { Length } \\
(\mathrm{Km})\end{array}$ & Number of Circuits) \\
\hline 1. & Ikot Ekpene T.S - Ibom G.S & 2.4 & \multirow{23}{*}{ Double } \\
\hline 2. & Ikot Ekpene T.S - Afam G.S & 107.8 & \\
\hline 3. & Aba G.S - Alaoji T.S & 14 & \\
\hline 4. & Ibom G.S - Eket T.S & 37.4 & \\
\hline 5. & Ikot Ekpene T.S - Calabar G.S & 71 & \\
\hline 6. & Egbema T.S - Owerri T.S & 5 & \\
\hline 7. & Eket T.S - Itu T.S & 75 & \\
\hline 8. & Itu T.S - Aba T.S & 74.7 & \\
\hline 9. & Adiabor T.S - Itu T.S & 10 & \\
\hline 10 & Omotosho G.S - Ajah T.S & 145 & \\
\hline 11. & Kaduna T.S - Jos T.S & 217.5 & \\
\hline 12. & Birni - Kebbi T.S - Sokoto T.S & 162 & \\
\hline 13. & Sokoto T.S - Kastina T.S & 162.6 & \\
\hline 14. & Kastina T.S - Kano G.S & 171 & \\
\hline 15. & Kano G.S - Damaturu T.S & 429.9 & \\
\hline 16. & Maiduguri T.S - Yola T.S & 165 & \\
\hline 17. & Gwagwalada T.S - Lafia T.S & 223.2 & \\
\hline 18. & Gombe T.S - Dadinkowa T.S & 368.2 & \\
\hline 19. & Makurdi T.S - Mambilla G.S & 354 & \\
\hline 20. & Mambilla G.S - Jalingo T.S & 179 & \\
\hline 21. & Zungeru GS - Gwagwalada T.S & 181.4 & \\
\hline 22. & Yola T.S - Kiri G.S & 5 & \\
\hline 23. & Yola T.S - Jalingo G.S & 165 & \\
\hline
\end{tabular}


Power Flow Analysis of the Enhanced Proposed 330kV Transmission Network of the Nigeria Grid

TABLE 4. POWER FLOW RESULT OF THE ENHANCED NETWORK

\begin{tabular}{|c|c|c|c|c|c|c|c|c|c|c|c|c|c|}
\hline No. & $\begin{array}{l}\text { Bus } \\
\text { ID }\end{array}$ & $\begin{array}{l}\mathrm{V} \\
\text { (p.u) }\end{array}$ & $\begin{array}{l}\text { Pgen } \\
(\mathrm{MW})\end{array}$ & $\begin{array}{l}\text { Qgen } \\
\text { (Mvar) }\end{array}$ & $\begin{array}{l}\text { Pload } \\
\text { (MW) }\end{array}$ & $\begin{array}{l}\text { Qload } \\
\text { (Mvar) }\end{array}$ & No. & $\begin{array}{l}\text { Bus } \\
\text { ID }\end{array}$ & $\begin{array}{l}\mathrm{V} \\
\text { (p.u) }\end{array}$ & $\begin{array}{l}\text { Pgen } \\
(\mathrm{MW})\end{array}$ & $\begin{array}{l}\text { Qgen } \\
\text { (Mvar) }\end{array}$ & $\begin{array}{l}\text { Pload } \\
\text { (MW) }\end{array}$ & $\begin{array}{l}\text { Qload } \\
\text { (Mvar) }\end{array}$ \\
\hline 1. & Aba T.S & 1.02 & 0 & 0 & 0 & 0 & 40. & Jebba T.S & 1 & 0 & 0 & 622.5 & 385.79 \\
\hline 2. & Adiabor T.S & 1.02 & 0 & 0 & 0 & 0 & 41. & Jos T.S & 0.96 & 0 & 0 & 413.9 & 256.54 \\
\hline 3. & Aes GS & 0.98 & 236.27 & 160.26 & 121.17 & 75.09 & 42. & Kaduna T.S & 1.01 & 0 & 0 & 55.84 & 34.604 \\
\hline 4. & Afam GS & 1.03 & 597.81 & 234 & 285.75 & 177.1 & 43. & Kainji G.S & 1.02 & 672.4 & 332.04 & 509.9 & 316.03 \\
\hline 5. & Ahoda T.S & 1.03 & 0 & 0 & 0 & 0 & 44. & Kainji T.S & 1.02 & 0 & 0 & 0 & 0 \\
\hline 6. & Aiyede T.S & 0.96 & 0 & 0 & 167.37 & 103.7 & 45 & Kano G.S & 1.01 & 496.64 & 227.16 & 562.3 & 348.5 \\
\hline 7. & Aja T.S & 0.97 & 0 & 0 & 34.321 & 21.27 & 46. & Kastina T.S & 0.95 & 0 & 0 & 211.5 & 131.09 \\
\hline 8. & Ajaokuta T.S & 1.04 & 0 & 0 & 194.9 & 120.8 & 47. & Katampe T.S & 0.96 & 0 & 0 & 384.5 & 238.27 \\
\hline 9. & Akangba T.S & 0.88 & 0 & 0 & 157.59 & 97.67 & 48 & Kiri GS & 0.95 & 27.717 & 16.023 & 326.7 & 202.47 \\
\hline 10. & Aladja T.S & 0.96 & 0 & 0 & 330.67 & 204.9 & 49. & Lafia T.S & 0.96 & 0 & 0 & 202.5 & 125.47 \\
\hline 11. & Alagbon T.S & 0.97 & 0 & 0 & 34.295 & 21.25 & 50. & Lekki T.S & 0.97 & 0 & 0 & 0 & 0 \\
\hline 12. & Alaoji GS & 1.04 & 862.03 & 393.58 & 197.8 & 122.6 & 51. & Lokoja T.S & 1.05 & 0 & 0 & 0 & 0 \\
\hline 13. & Alaoji T.S & 1.02 & 0 & 0 & 564.85 & 350.1 & 52. & Maiduguri T.S & 0.97 & 0 & 0 & 103.3 & 64.032 \\
\hline 14. & Asaba T.S & 1.02 & 0 & 0 & 0 & 0 & 53. & Makurdi T.S & 1.03 & 0 & 0 & 193.7 & 120.05 \\
\hline 15. & Asco G.S & 1.02 & 39.356 & 17.783 & 190.05 & 117.8 & 54. & Mambilla G.S & 1.05 & 2637.7 & 1146.2 & 1388 & 860.39 \\
\hline 16. & Azura G.S & 1.04 & 384.75 & 167.02 & 196.87 & 122 & 55 & \begin{tabular}{|c|} 
New Haven \\
T.S \\
\end{tabular} & 1.02 & 0 & 0 & 202.8 & 125.69 \\
\hline 17. & Benin T.S & 1.04 & 0 & 0 & 194.9 & 120.8 & 56. & Odupkani G.S & 1.02 & 212.78 & 131.01 & 282.4 & 175.02 \\
\hline 18. & $\begin{array}{c}\text { Birni-Kebbi } \\
\text { T.S } \\
\end{array}$ & 0.98 & 0 & 0 & 121.67 & 75.4 & 57. & Okaero T.S & 0.92 & 0 & 0 & 230.9 & 143.09 \\
\hline 19. & Calabar GS & 1.07 & 543.2 & 333.79 & 257.58 & 159.6 & 58. & Okpia G.S & 1.04 & 362.67 & 190.57 & 19.45 & 12.054 \\
\hline 20 & Dadinkowa T.S & 0.8 & 0 & 0 & 230.46 & 142.8 & 59. & $\begin{array}{c}\text { Olorunsogo } \\
\text { G.S }\end{array}$ & 1.04 & 935.13 & 476.87 & 0 & 0 \\
\hline 21. & Damaturu T.S & 1.04 & 0 & 0 & 0 & 0 & 60. & Omoku G.S & 1.04 & 350.67 & 187.75 & 388.6 & 240.86 \\
\hline 22. & Delta GS & 1 & 787.96 & 339.8 & 216.94 & 134.4 & 61. & Omotosho G.S & 0.98 & 710.35 & 416.06 & 0 & 0 \\
\hline 23. & Egbema GS & 1.04 & 316.68 & 135.5 & 195.12 & 120.9 & 62. & Onitsha T.S & 1.01 & 0 & 0 & 520.5 & 322.59 \\
\hline 24. & Egbin GS & 0.97 & 1065.5 & 589.27 & 170.2 & 105.5 & 63. & Oshogbo T.S & 0.97 & 0 & 0 & 255.3 & 158.21 \\
\hline 25. & Eket T.S & 1.02 & 0 & 0 & 0 & 0 & 64. & Owerri T.S & 1.03 & 0 & 0 & 0 & 0 \\
\hline 26. & Ganmo T.S & 0.96 & 0 & 0 & 199.39 & 123.6 & 65 & \begin{tabular}{|c|} 
Paras energy \\
G.S \\
\end{tabular} & 0.99 & 44.041 & 29.013 & 0 & 0 \\
\hline 27. & Gbarain GS & 1.01 & 109.83 & 57.148 & 184.06 & 114.1 & 66. & PH main T.S & 1.03 & 0 & 0 & 0 & 0 \\
\hline 28 & Geregu GS & 1.05 & 763.15 & 355.23 & 277.14 & 171.8 & 67. & Sagamu T.S & 0.99 & 0 & 0 & 0 & 0 \\
\hline 29. & Gombe T.S & 0.95 & 0 & 0 & 164.18 & 101.8 & 68. & Sapele GS & 1.05 & 1313.9 & 604.36 & 503.5 & 312.06 \\
\hline 30. & \begin{tabular}{|c|} 
Gwagwalada \\
T.S \\
\end{tabular} & 1.02 & 0 & 0 & 0 & 0 & 69. & Shiroro GS & 1.03 & 537.43 & 204.05 & 270.8 & 167.83 \\
\hline 31. & Ibom GS & 1.03 & 76.176 & 39.934 & 0 & 0 & 70. & Sokoto T.S & 0.93 & 0 & 0 & 297.7 & 184.48 \\
\hline 32. & Ihovor GS & 1.04 & 356.22 & 151.22 & 197.44 & 122.4 & 71. & $\begin{array}{c}\text { Trans amadi } \\
\text { G.S } \\
\end{array}$ & 1.02 & 158.18 & 72.989 & 190.1 & 117.8 \\
\hline 33. & Ikeja West T.S & 0.89 & 0 & 0 & 1141.8 & 707.6 & 72. & Ugwuaji T.S & 1.02 & 0 & 0 & 0 & 0 \\
\hline 34. & Ikorodu T.S & 0.99 & 0 & 0 & 0 & 0 & 73. & Yenegoa T.S & 1 & 0 & 0 & 180.9 & 112.14 \\
\hline 35. & $\begin{array}{c}\text { IkotEpkene } \\
\text { T.S } \\
\end{array}$ & 1.03 & 0 & 0 & 286.09 & 177.3 & 74. & Yola T.S & 0.95 & 0 & 0 & 32.84 & 20.354 \\
\hline 36. & Itobe GS & 1.05 & 1000.5 & 503.29 & 502.33 & 311.3 & 75. & Zamfara GS & 1 & 86.218 & 45.584 & 273.8 & 169.71 \\
\hline 37. & Itu T.S & 1.01 & 0 & 0 & 371.4 & 230.2 & 76. & Zungeru GS & 1.04 & 592.46 & 246.16 & 393.9 & 244.09 \\
\hline 38. & Jalingo T.S & 0.98 & 0 & 0 & 172.87 & 107.1 & \multirow{2}{*}{\multicolumn{3}{|c|}{ Total }} & \multirow{2}{*}{16747} & \multirow{2}{*}{8025.3} & \multirow{2}{*}{16400} & \multirow{2}{*}{10164} \\
\hline 39. & Jebba G.S & 1 & 469.11 & 221.64 & 22.39 & 13.877 & & & & & & & \\
\hline
\end{tabular}

Mehran University Research Journal of Engineering \& Technology, Volume 38, No. 4, October, 2019 [p-ISSN: 0254-7821, e-ISSN: 2413-7219] 
Power Flow Analysis of the Enhanced Proposed 330kV Transmission Network of the Nigeria Grid

TABLE 5. LINE FLOW RESULT OF THE ENHANCED NETWORK

\begin{tabular}{|c|c|c|c|c|c|c|c|c|c|}
\hline No. & From Bus & To Bus & $\begin{array}{c}\text { P Losses } \\
\text { (MW) }\end{array}$ & $\begin{array}{c}\text { Q Losses } \\
\text { (Mvar) }\end{array}$ & No. & From Bus & To Bus & $\begin{array}{c}\text { P Losses } \\
\text { (MW) }\end{array}$ & $\begin{array}{c}\text { Q Losses } \\
\text { (Mvar) }\end{array}$ \\
\hline 1. & Aba T.S & Alaoji T.S & 0.01 & -9.92 & 48. & Ikeja West T.S & Okaero T.S & 6.63 & 11.11 \\
\hline 2. & Aba T.S & Itu T.S & 0.09 & -52.43 & 49. & Ikorodu T.S & Sagamu T.S & 0.04 & -0.89 \\
\hline 3. & Adiabor T.S & Odupkani G.S & 0.43 & -5.15 & 50. & IkotEpkene T.S & Afam GS & 0.03 & -1.01 \\
\hline 4. & Adiabor T.S & Itu T.S & 0.44 & -5.08 & 51. & IkotEpkene T.S & Ugwuaji T.S & 4.03 & -106.67 \\
\hline 5. & Afam GS & PH main T.S & 0.11 & -5.27 & 52. & IkotEpkene T.S & Alaoji T.S & 0.09 & -41.14 \\
\hline 6. & Ahoda T.S & Gbarain GS & 0.53 & -59.62 & 53. & Itobe GS & Lokoja T.S & 0.78 & -4.08 \\
\hline 7. & Aiyede T.S & Olorunsogo GS & 6.54 & -22.68 & 54. & Itu T.S & Eket T.S & 0.23 & -52.3 \\
\hline 8. & Aja T.S & Lekki T.S & 0 & -3.57 & 55. & Jalingo T.S & Yola T.S & 8.15 & -68.28 \\
\hline 9. & Aja T.S & Alagbon T.S & 0 & -13.61 & 56. & Jebba G.S & Jebba T.S & 0.14 & -4.88 \\
\hline 10 & Ajaokuta T.S & Benin T.S & 0.85 & -140.15 & 57. & Jebba T.S & Ganmo T.S & 3.21 & -7.31 \\
\hline 11. & Ajaokuta T.S & Geregu GS & 1.15 & -125.08 & 58. & Jebba T.S & Oshogbo T.S & 2.22 & -94.95 \\
\hline 12. & Akangba T.S & Ikeja West T.S & 0.42 & -7.81 & 59. & Jebba T.S & Shiroro GS & 0.36 & -171.83 \\
\hline 13. & Aladja T.S & Delta GS & 5.33 & 15.09 & 60. & Jos T.S & Makurdi T.S & 5.59 & -169.31 \\
\hline 14. & Alagbon T.S & Lekki T.S & 0 & -15.89 & 61. & Kaduna T.S & Shiroro GS & 11.77 & -16.65 \\
\hline 15. & Alaoji GS & Alaoji T.S & 6.41 & -8.44 & 62. & Kaduna T.S & Jos T.S & 7.8 & -110.55 \\
\hline 16. & Alaoji T.S & Onitsha T.S & 0.32 & -44.98 & 63. & Kainji T.S & Kainji G.S & 0.03 & -0.2 \\
\hline 17. & Alaoji T.S & Owerri T.S & 0.11 & -54.87 & 64. & Kainji T.S & Jebba T.S & 1.95 & -48.33 \\
\hline 18. & Alaoji T.S & Afam GS & 0.19 & -17.08 & 65. & Kano G.S & Damaturu T.S & 7.18 & -278.14 \\
\hline 19. & Asaba T.S & Benin T.S & 1.53 & -93.06 & 66. & Kano G.S & Kaduna T.S & 0.12 & -76.64 \\
\hline 20 & Asaba T.S & Onitsha T.S & 0.95 & -42.34 & 67. & Kastina T.S & Sokoto T.S & 1.29 & -92.41 \\
\hline 21. & Asco GS & Ajaokuta T.S & 0.56 & -33.97 & 68. & Kastina T.S & Kano GS & 10.87 & -63 \\
\hline 22. & Azura GS & Benin T.S & 0.56 & -30.91 & 69. & Kiri GS & Yola T.S & 0.12 & -2.57 \\
\hline 23. & Benin T.S & Sapele G.S & 4.81 & -16.42 & 70. & Lafia T.S & Gwagwalada T.S & 5.51 & -126.59 \\
\hline 24. & Benin T.S & Delta GS & 0.72 & -33.1 & 71. & Lokoja T.S & Ajaokuta T.S & 1.37 & -155.09 \\
\hline 25. & Benin T.S & Egbin G.S & 1.41 & -65.62 & 72. & Maiduguri T.S & Yola T.S & 1.21 & -99.75 \\
\hline 26. & Benin T.S & Omotosho GS & 1.75 & -32.04 & 73. & Makurdi T.S & Ugwuaji T.S & 2.01 & -181.61 \\
\hline 27. & Birni-Kebbi T.S & Kainji T.S & 7.49 & -67.38 & 74. & Mambilla GS & Makurdi T.S & 12.48 & -205.98 \\
\hline 28. & Calabar GS & IkotEpkene T.S & 4.21 & -34.38 & 75. & Mambilla GS & Jalingo T.S & 21.93 & -26.2 \\
\hline 29. & Dadinkowa T.S & Gombe T.S & 17.28 & -116.26 & 76. & New Haven T.S & Onitsha T.S & 1.39 & -25.92 \\
\hline 30. & Damaturu T.S & Maiduguri T.S & 0.8 & -83.39 & 77. & Odupkani G.S & IkotEpkene T.S & 0.05 & -63.26 \\
\hline 31. & Egbin GS & Aja T.S & 0.15 & -8.4 & 78. & Okaero T.S & Egbin G.S & 13.25 & 39.67 \\
\hline 32. & Egbin GS & Aes GS & 0.68 & -9.99 & 79. & Okpia GS & Onitsha T.S & 6.08 & -13.4 \\
\hline 33. & Egbin GS & Ikorodu T.S & 1.52 & -27.69 & 80. & Omoku GS & PH main T.S & 1.59 & -53.24 \\
\hline 34. & Ganmo T.S & Oshogbo T.S & 0.08 & -26.12 & 81. & Omotosho GS & Aja T.S & 0.44 & -11.8 \\
\hline 35. & Gbarain GS & Yenegoa T.S & 0.57 & -14.68 & 82. & Oshogbo T.S & Aiyede T.S & 0.06 & -36.1 \\
\hline 36. & Gombe T.S & Yola T.S & 2.5 & -59.88 & 83. & Oshogbo T.S & Ihovor GS & 3.83 & -65.97 \\
\hline 37. & Gombe T.S & Kaduna T.S & 10.54 & -32.78 & 84. & Owerri T.S & Ahoda T.S & 0.02 & -3.52 \\
\hline 38. & Gombe T.S & Damaturu T.S & 4.68 & -30.92 & 85. & Owerri T.S & Egbema G.S & 0.79 & -73.34 \\
\hline 39. & Gwagwalada T.S & Shiroro GS & 0.03 & -49.91 & 86. & Paras energy G.S & Sagamu T.S & 0.11 & -2.23 \\
\hline 40. & Gwagwalada T.S & Lokoja T.S & 5.36 & -76.45 & 87. & Sapele GS & Delta GS & 3.4 & -16.56 \\
\hline 41. & Gwagwalada T.S & Katampe T.S & 6.34 & 9.71 & 88. & Shiroro GS & Katampe T.S & 3.18 & -32.42 \\
\hline 42. & Ibom GS & Eket T.S & 0.06 & -26.7 & 89. & Sokoto T.S & Birni-Kebbi T.S & 3.85 & -83.86 \\
\hline 43. & Ibom GS & IkotEpkene T.S & 0.04 & -1.55 & 90. & Trans amadi GS & PH main T.S & 0.02 & -5.67 \\
\hline 44. & Ihovor GS & Benin T.S & 0.15 & -27.74 & 91. & Ugwuaji T.S & New Haven T.S & 0.12 & -4.12 \\
\hline 45. & Ikeja West T.S & Olorunsogo GS & 13.9 & 17.81 & 92. & Zamfara G.S & Birni-Kebbi T.S & 4.58 & -185.57 \\
\hline 46. & Ikeja West T.S & Egbin G.S & 8.78 & 22.76 & 93. & Zungeru GS & Gwagwalada T.S & 3.41 & -117.43 \\
\hline 47. & Ikeja West T.S & Omotosho GS & 11.56 & 35.94 & Total & & 252.25 & & \\
\hline
\end{tabular}

Mehran University Research Journal of Engineering \& Technology, Volume 38, No. 4, October, 2019 [p-ISSN: 0254-7821, e-ISSN: 2413-7219] 


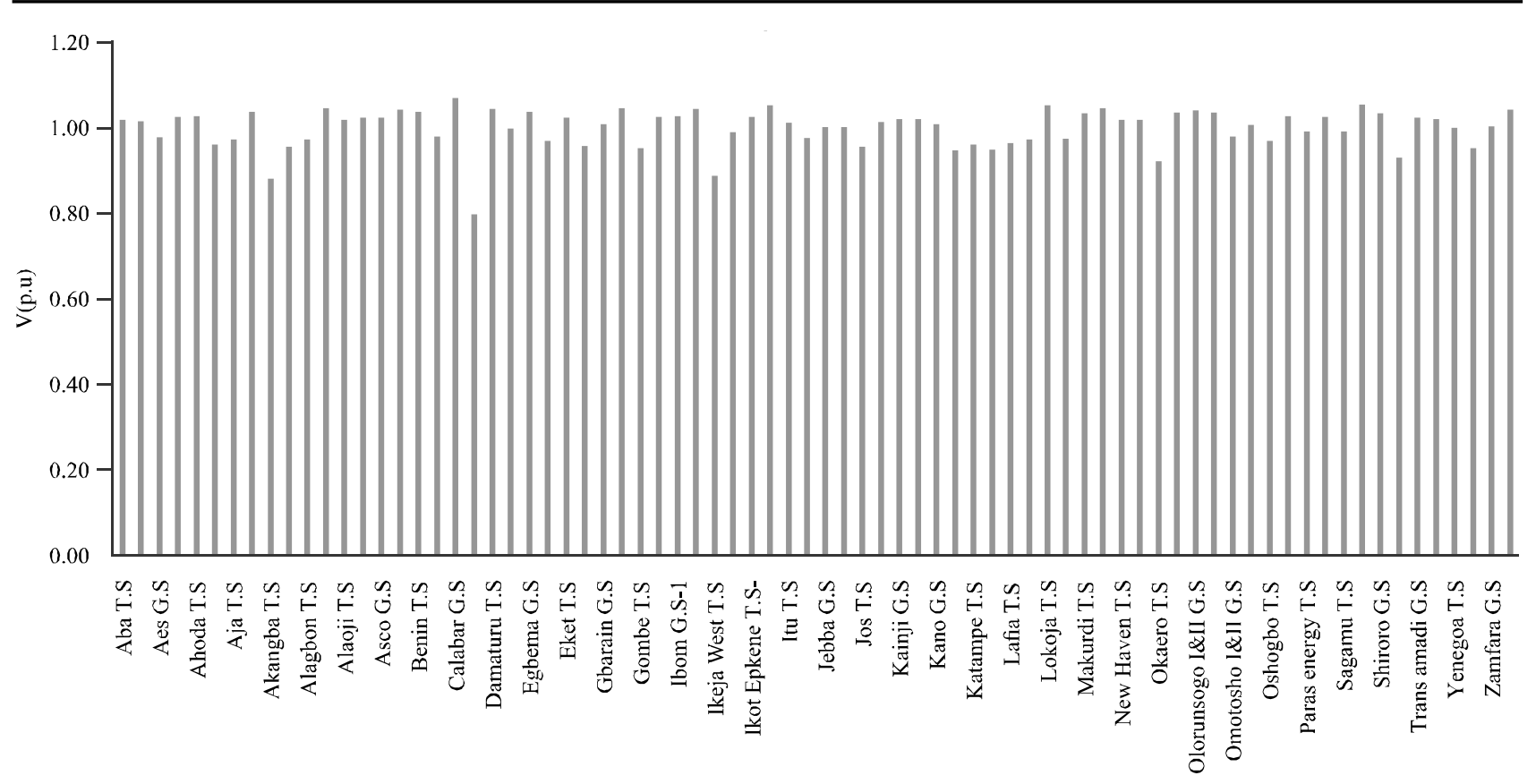

FIG. 1. VOLTAGE PROFILE OF THE ENHANCED NETWORK

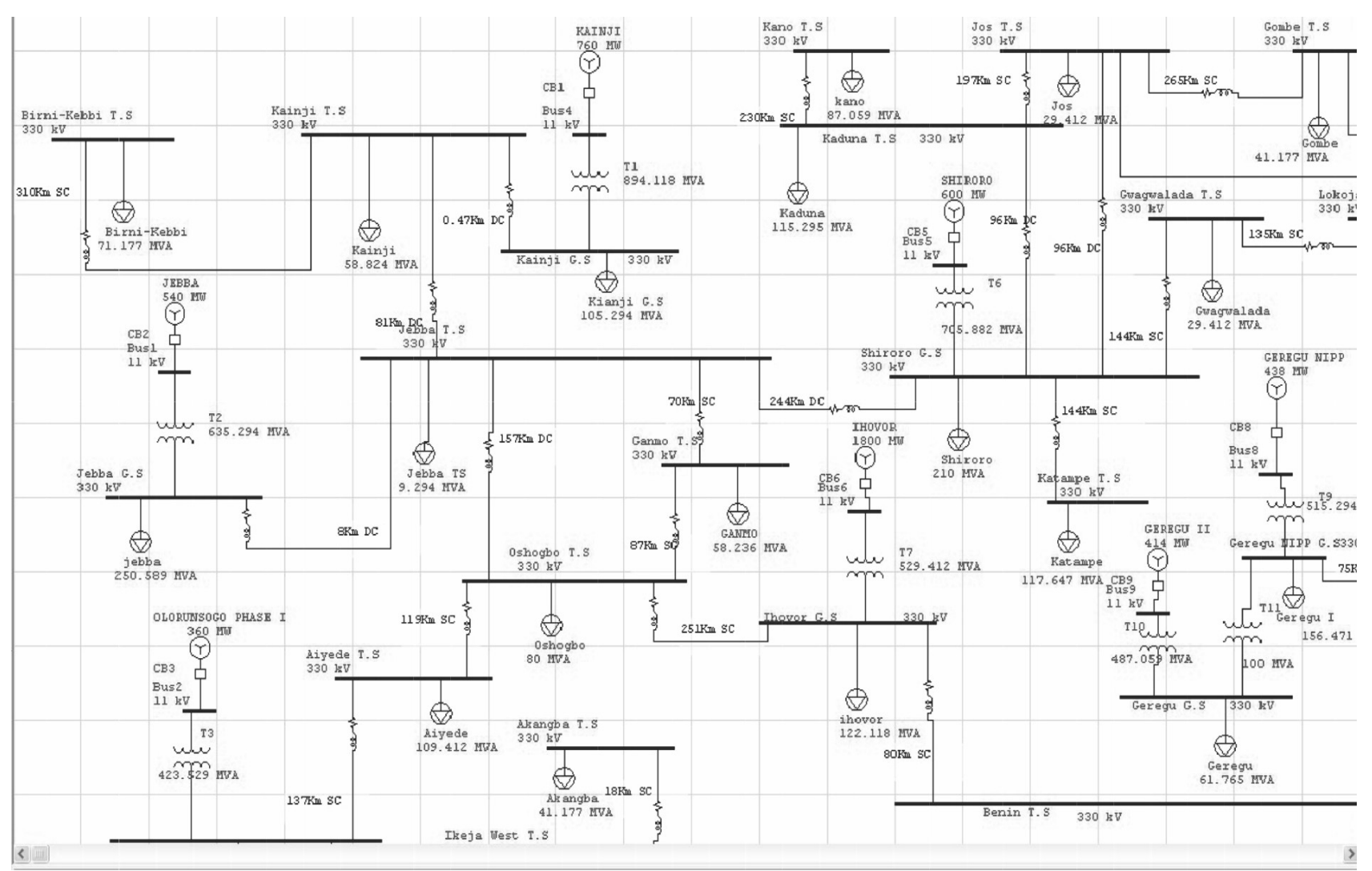

FIG. 2. SECTION OF THE LOAD FLOW SIMULATION DIAGRAM MODELED ON ETAP 12.6 EDIT MODE 


\section{CONCLUSION}

In this work, the system profile analysis revealed that the existing network evacuated more power when more power station and transmission lines were added for the purpose of system expansion. The maximum power the network evacuated was 16,746.859MW and 8,025.291MVar with loading of 16,400.067MW and 10,163.847MVar. Nigeria grid network can only evacuate between 1,000 and 4,000MW due to inefficiency, unreliability, not properly ringed network, high losses and power station breakdown. From the analysis, more generating stations and transmission lines should be built and added to the network and the single circuit lines should be upgraded to double circuit lines in order to boost generation and transmission, while control devices should be used in high and low voltage areas.

\section{ACKNOWLEDGEMENT}

The authors are grateful to Prof. SA Ike, Department Electrical/Electronic Engineering, Faculty of Engineering, University of Benin, Benin City, for his assistance and supervision throughout the period this work was done.

\section{REFERENCES}

[1] Mathaios, P., Dimitris, N.T.,Pierluigi, M., and Nikos, D.H, "Power Systems Resilience Assessment: Hardening and Smart Operational Enhancement Strategies", Proceedings of IEEE, Volume 105, No. 7 , pp 1-11, 2017.

[2] Emmanuel, U.O., Samuel, N.N., and Gerald, K.I., "Electric Power Transmission Enhancement: A Case of Nigerian Electric Power Grid", American Journal of Electrical and Electronic Engineering, Volume 4, No. 1, pp. 33-39, 2016.
[3] Oshevire, P., Oladimeji, T., and Onohaebi, S.,"Smart Grid Technology and its Possible Applications to the Nigeria 330 kV Power System”, Smart Grid \& Renewable Energy, Volume 4, pp. 391-397, 2013.

[4] Sunday, O.O., and Friday, O.O., "Empirical Modeling of Power Losses as a Function of Line Loadings and Lengths in the Nigerian $330 \mathrm{kV}$ Transmission Lines", International Journal of Academic Research, Volume 2, pp. 47-53, 2010 .

[5] Utazi, D.N., and Ujam, A.T., "The Need to Expand and Modernize the Electricity Transmission Infrastructure in Nigeria", International Journal of Engineering Trends and Technology, Volume 12, No. 8, pp. 411 413, 2014

[6] Oriaifo, A.P., and Orukpe, P.E., “Analysis of Voltage Collapse in Power Systems", Journal of Electrical and Electronic Engineering, Volume 13 No. 1, pp. 16-24, Nigeria, 2016.

Hasham, K., and Mohammad, A.C., "Performance Enhancement of Low Voltage Distribution Network in Developing Countries using Hybrid Rehabilitation Technique", Mehran University Research Journal of Engineering \& Technology, Volume 37, No. 3, pp. 493512, Jamshoro, Pakistan, July 2018.

[8] Igbinovia, S.O., and Orukpe, P.E., "Rural Electrification: The Propelling Force for Rural Development of Edo State, Nigeria", Journal of Energy in Southern Africa, Volume 18, No. 3, pp. 18-26, 2007.

[9] Onohaebi, O.S., "Power Outage in the Nigeria Transmission Grid”, Research Journal of Applied Sciences, Volume 4, No. 1, pp. 1-9, 2009.

[10] Babatunde R.F., "Power Sector Reforms-Challenges and the Way Forward", 2017http://thenewsnigeria.com.ng/ $2017 / 07 /$ power-sector-reforchallengesandthewayforward/ Accessed:20/4/19.

[11] The Nation, "Electricity: Challenges and Prospects", 2017http:/www.nigeriaelectricityhub.com/2017/03/10/ electricity-challenges-and-prospects/.Accessed: 25/4/19 
[12] Abubakar, S.S., "The Way Forward for Electricity Supply in Nigeria", Nigerian National Committee of the World Energy Council, 2015 http:// voices.nationalgeographic.com/2015/10/20/the-wayforward-for-electricity-supply-in-nigeria/. Accessed: 25 th April, 2019.

[13] Onah, J.N., "Contingency Evaluation of the Nigerian 330kV Transmission Grid”, University of Nigeria, Nsukka, M.E. Thesis, pp. 33-39, 2015.
[14] Transmission Company of Nigeria, "Transmission Plans (2013-2017) and Evacuation of Niger Delta Power Holding Company (NDPHC) Power Plants Generation”, Presidential Task Force on Power Summit, Abuja. pp. 4-19, 2013.

[15] Agbontaen, F.O., and Ike, S.A., "Loadability Analysis of the Existing $330 \mathrm{kV}$ Transmission Network of the Nigerian Electricity Grid”, Nigerian Research Journal of Engineering and Environmental Sciences, Volume 2 No. 2, pp. 416-423, 2017. 